\title{
SOFT CLOSURE OPERATORS, SOFT TOPOLOGIES AND SOFT QUASI-UNIFORMITIES
}

\author{
Yong Chan Kim \\ Department of Mathematics \\ Gangneung-Wonju University \\ Gangneung, Gangwondo, 210-702, KOREA
}

\begin{abstract}
In this paper, we study the notions of soft closure operators in complete residuated lattices. We investigate the relations among soft topologies, soft closure operators and soft $L$-quasi-uniformities in complete residuated lattices. We give their examples.
\end{abstract}

AMS Subject Classification: 03E72, 06A15, 06F07, 54A05

Key Words: complete residuated lattice, soft quasi-uniformities, soft topologies, soft closure operators, uniformly continuous soft map, continuous soft maps

\section{Introduction}

Hájek [4] introduced a complete residuated lattice which is an algebraic structure for many valued logic. It is an important mathematical tool for algebraic structures [5,7-9]. Recently, Molodtsov [11] introduced the soft set as a mathematical tool for dealing information as the uncertainty of data in engineering, physics, computer sciences and many other diverse field. Presently, the soft set theory is making progress rapidly $[1,3]$. Pawlak's rough set $[12,13]$ can be viewed as a special case of soft rough sets [3]. The topological structures of soft sets have been developed by many researchers [2,7-9,14-17].

Ko [7] introduced a fuzzy soft $F: A \rightarrow L^{U}$ as an extension as the soft $F$ : $A \rightarrow P(U)$ where $L$ is a complete residuated lattice. Ko [7-9] introduced the soft topological structures, $L$-fuzzy quasi-uniformities and soft $L$-fuzzy topogenous orders in complete residuated lattices.

Received: April 10, 2016

Published: April 22, 2016 (c) 2016 Academic Publications, Ltd.

url: www.acadpubl.eu 
In this paper, we study the notions of soft closure operators in complete residuated lattices. We investigate the relations among soft topologies, soft closure operators and soft $L$-quasi-uniformities in complete residuated lattices. We give their examples.

\section{Preliminaries}

Definition 1. [4,5] An algebra $(L, \wedge, \vee, \odot, \rightarrow, 0,1)$ is called a complete residuated lattice if it satisfies the following conditions:

(C1) $L=(L, \leq, \vee, \wedge, 1,0)$ is a complete lattice with the greatest element 1 and the least element 0 ;

(C2) $(L, \odot, 1)$ is a commutative monoid;

(C3) $x \odot y \leq z$ iff $x \leq y \rightarrow z$ for $x, y, z \in L$.

In this paper, we assume that $(L, \leq, \odot, \rightarrow)$ is a complete residuated lattice.

Lemma 2. [4,5] For each $x, y, z, x_{i}, y_{i}, w \in L$, we have the following properties.

(1) $1 \rightarrow x=x, 0 \odot x=0$,

(2) If $y \leq z$, then $x \odot y \leq x \odot z, x \rightarrow y \leq x \rightarrow z$ and $z \rightarrow x \leq y \rightarrow x$,

(3) $x \odot y \leq x \wedge y \leq x \vee y$,

(4) $x \odot\left(\bigvee_{i} y_{i}\right)=\bigvee_{i}\left(x \odot y_{i}\right)$,

(5) $x \rightarrow\left(\bigwedge_{i} y_{i}\right)=\bigwedge_{i}\left(x \rightarrow y_{i}\right)$,

(6) $\left(\bigvee_{i} x_{i}\right) \rightarrow y=\bigwedge_{i}\left(x_{i} \rightarrow y\right)$,

(7) $x \rightarrow\left(\bigvee_{i} y_{i}\right) \geq \bigvee_{i}\left(x \rightarrow y_{i}\right)$,

(8) $\left(\bigwedge_{i} x_{i}\right) \rightarrow y \geq \bigvee_{i}\left(x_{i} \rightarrow y\right)$,

(9) $(x \odot y) \rightarrow z=x \rightarrow(y \rightarrow z)=y \rightarrow(x \rightarrow z)$,

(10) $x \odot(x \rightarrow y) \leq y$ and $x \rightarrow y \leq(y \rightarrow z) \rightarrow(x \rightarrow z)$,

(11) $(x \rightarrow y) \odot(z \rightarrow w) \leq(x \odot z) \rightarrow(y \odot w)$,

(12) $x \rightarrow y \leq(x \odot z) \rightarrow(y \odot z)$ and $(x \rightarrow y) \odot(y \rightarrow z) \leq x \rightarrow z$. 
Definition 3. [7-9] Let $X$ be an initial universe of objects and $E$ the set of parameters (attributes) in $X$. A pair $(F, A)$ is called a fuzzy soft set over $X$, where $A \subset E$ and $F: A \rightarrow L^{X}$ is a mapping. We denote $S(X, A)$ as the family of all fuzzy soft sets under the parameter $A$.

Definition 4. [7-9] Let $(F, A)$ and $(G, A)$ be two fuzzy soft sets over a common universe $X$.

(1) $(F, A)$ is a fuzzy soft subset of $(G, A)$, denoted by $(F, A) \leq(G, A)$ if $F(a) \leq G(a)$, for each $a \in A$.

(2) $(F, A) \wedge(G, A)=(F \wedge G, A)$ if $(F \wedge G)(a)=F(a) \wedge G(a)$ for each $a \in A$.

(3) $(F, A) \vee(G, A)=(F \vee G, A)$ if $(F \vee G)(a)=F(a) \vee G(a)$ for each $a \in A$

(4) $(F, A) \odot(G, A)=(F \odot G, A)$ if $(F \odot G)(a)=F(a) \odot G(a)$ for each $a \in A$.

(6) $\alpha \odot(F, A)=(\alpha \odot F, A)$ for each $\alpha \in L$.

Definition 5. [7-9] Let $S(X, A)$ and $S(Y, B)$ be the families of all fuzzy soft sets over $X$ and $Y$, respectively. The mapping $f_{\phi}: S(X, A) \rightarrow S(Y, B)$ is a soft mapping where $f: X \rightarrow Y$ and $\phi: A \rightarrow B$ are mappings.

(1) The image of $(F, A) \in S(X, A)$ under the mapping $f_{\phi}$ is denoted by $f_{\phi}((F, A))=\left(f_{\phi}(F), B\right)$, where

$$
f_{\phi}(F)(b)(y)= \begin{cases}\bigvee_{a \in \phi^{-1}(\{b\})} f^{\rightarrow}(F(a))(y), & \text { if } \phi^{-1}(\{b\}) \neq \emptyset \\ 0, & \text { otherwise }\end{cases}
$$

(2) The inverse image of $(G, B) \in S(Y, B)$ under the mapping $f_{\phi}$ is denoted by $f_{\phi}^{-1}((G, B))=\left(f_{\phi}^{-1}(G), A\right)$ where

$$
f_{\phi}^{-1}(G)(a)(x)=f^{\leftarrow}(G(\phi(a)))(x), \forall a \in A, x \in X .
$$

(3) The soft mapping $f_{\phi}: S(X, A) \rightarrow S(Y, B)$ is called injective (resp. surjective, bijective) if $f$ and $\phi$ are both injective (resp. surjective, bijective).

Lemma 6. [7-9] Let $f_{\phi}: S(X, A) \rightarrow S(Y, B)$ be a soft mapping. Then we have the following properties. For $(F, A),\left(F_{i}, A\right) \in S(X, A)$ and $(G, B),\left(G_{i}, B\right) \in$ $S(Y, B)$,

(1) $(G, B) \geq f_{\phi}\left(f_{\phi}^{-1}((G, B))\right)$ with equality if $f$ is surjective, 
(2) $(F, A) \leq f_{\phi}^{-1}\left(f_{\phi}((F, A))\right)$ with equality if $f$ is injective,

(3) $f_{\phi}^{-1}\left(\bigvee_{i \in I}\left(G_{i}, B\right)\right)=\bigvee_{i \in I} f_{\phi}^{-1}\left(\left(G_{i}, B\right)\right)$,

(4) $f_{\phi}^{-1}\left(\bigwedge_{i \in I}\left(G_{i}, B\right)\right)=\bigwedge_{i \in I} f_{\phi}^{-1}\left(\left(G_{i}, B\right)\right)$,

(5) $f_{\phi}\left(\bigvee_{i \in I}\left(F_{i}, A\right)\right)=\bigvee_{i \in I} f_{\phi}\left(\left(F_{i}, A\right)\right)$,

(6) $f_{\phi}\left(\bigwedge_{i \in I}\left(F_{i}, A\right)\right) \leq \bigwedge_{i \in I} f_{\phi}\left(\left(F_{i}, A\right)\right)$ with equality if $f$ is injective,

(7) $f_{\phi}^{-1}\left(\left(G_{1}, B\right) \odot\left(G_{2}, B\right)\right)=f_{\phi}^{-1}\left(\left(G_{1}, B\right)\right) \odot f_{\phi}^{-1}\left(\left(G_{2}, B\right)\right)$,

(8) $f_{\phi}\left(\left(F_{1}, A\right) \odot\left(F_{2}, A\right)\right) \leq f_{\phi}\left(\left(F_{1}, A\right)\right) \odot f_{\phi}\left(\left(F_{2}, A\right)\right)$ with equality if $f$ is injective.

Definition 7. [7-9] A map $\tau \subset S(X, A)$ is called a soft topology on $X$ if it satisfies the following conditions.

$(\mathrm{ST} 1)\left(0_{X}, A\right),\left(1_{X}, A\right) \in \tau$, where $0_{X}(a)(x)=0,1_{X}(a)(x)=1$ for all $a \in$ $A, x \in X$,

(ST2) If $(F, A),(G, A) \in \tau$, then $(F, A) \odot(G, A) \in \tau$,

(ST3) If $\left(F_{i}, A\right) \in \tau$ for each $i \in I, \bigvee_{i \in I}\left(F_{i}, A\right) \in \tau$.

The triple $(X, A, \tau)$ is called a soft topological space.

Let $\left(X, A, \tau_{X}\right)$ and $\left(Y, B, \tau_{Y}\right)$ be soft topological spaces. A soft map $f_{\phi}$ : $\left(X, A, \tau_{X}\right) \rightarrow\left(Y, B, \tau_{Y}\right)$ is called a continuous soft map if $f_{\phi}^{-1}(G, B) \in \tau_{X}$, for all $(G, B) \in \tau_{Y}$.

Definition 8. [7-9] A subset $\mathbf{U} \subset S(X \times X, A)$ is called a soft $L$-quasiuniformity on $X$ iff it satisfies the properties.

(SU1) $\left(1_{X \times X}, A\right) \in \mathbf{U}$.

(SU2) If $(V, A) \leq(U, A)$ and $(V, A) \in \mathbf{U}$, then $(U, A) \in \mathbf{U}$.

(SU3) For every $(U, A),(V, A) \in \mathbf{U},(U, A) \odot(V, A) \in \mathbf{U}$.

(SU4) If $(U, A) \in \mathbf{U}$ then $\left(1_{\triangle}, A\right) \leq(U, A)$ where

$$
1_{\triangle}(a)(x, y)= \begin{cases}1, & \text { if } x=y \\ 0, & \text { if } x \neq y\end{cases}
$$

(SU5) For every $(U, A) \in \mathbf{U}$, there exists $(V, A) \in \mathbf{U}$ such that $(V, A) \circ$ $(V, A) \leq(U, A)$ where

$$
\begin{aligned}
& ((V, A) \circ(V, A))(a)(x, y)=(V(a) \circ V(a))(x, y) \\
& =\bigvee_{z \in X}(V(a)(z, x) \odot V(a)(x, y)), \quad \forall x, y \in X, a \in A
\end{aligned}
$$


The triple $(X, A, \mathbf{U})$ is called a soft $L$-quasi-uniform space.

Let $\left(X, A, \mathbf{U}_{X}\right)$ and $\left(Y, B, \mathbf{U}_{Y}\right)$ be soft quasi-uniform spaces. A soft map $f_{\phi}:\left(X, A, \mathbf{U}_{X}\right) \rightarrow\left(Y, B, \mathbf{U}_{Y}\right)$ is called an uniformly continuous soft map if $(f \times f)_{\phi}^{-1}(V, B) \in \mathbf{U}_{X}$, for all $(V, B) \in \mathbf{U}_{Y}$.

\section{Soft Closure Operators, Soft Topologies and Soft Quasi-Uniformities}

Definition 9. A mapping $c l: S(X, A) \rightarrow S(X, A)$ is called a soft closure operator if it satisfies the following conditions:

(C1) $\operatorname{cl}\left(0_{X}, A\right)=\left(0_{X}, A\right)$,

(C2) $\operatorname{cl}(F, A) \geq(F, A)$,

(C3) If $(F, A) \leq(G, A)$, then $\operatorname{cl}(F, A) \leq \operatorname{cl}(G, A)$,

(C4) $\operatorname{cl}(\operatorname{cl}(F, A))=(F, A)$,

(C5) $\operatorname{cl}((F, A) \odot(G, A)) \leq \operatorname{cl}(F, A) \odot \operatorname{cl}(G, A)$.

The triple $(X, A, c l)$ is called a soft closure space.

Let $\left(X, A, c l_{X}\right)$ and $\left(Y, B, c l_{Y}\right)$ be soft closure spaces and $f_{\phi}:(X, A) \rightarrow$ $(Y, B)$ be a map. Then $f_{\phi}$ is called a closure soft map if, for each $(F, A) \in$ $S(X, A)$

$$
c l_{Y}\left(f_{\phi}(F, A)\right) \geq f_{\phi}\left(c l_{X}(F, A)\right) .
$$

Theorem 10. Let $(X, A, \mathbf{U})$ be a soft quasi-uniform space. Define $c l_{\mathbf{U}}^{r}, c l_{\mathbf{U}}^{l}$ : $S(X, A) \rightarrow S(X, A)$ as follows

$$
\begin{aligned}
& c l_{\mathbf{U}}^{r}(F, A)(y)=\bigwedge_{(U, A) \in \mathbf{U}}\left(\bigvee_{x \in X}(U, A)(y, x) \odot(F, A)(x)\right), \\
& c l_{\mathbf{U}}^{l}(F, A)(y)=\bigwedge_{(U, A) \in \mathbf{U}}\left(\bigvee_{x \in X}(U, A)(x, y) \odot(F, A)(x)\right) .
\end{aligned}
$$

Then, for $c l \in\left\{c l_{\mathbf{U}}^{r}, c l_{\mathbf{U}}^{l}\right\}$, we have following properties:

(1) $\operatorname{cl}\left(0_{X}, A\right)=\left(0_{X}, A\right)$ and $\operatorname{cl}(F, A) \leq \operatorname{cl}(G, A)$ for $(F, A) \leq(G, A)$.

(2) $(F, A) \leq c l(F, A)$.

(3) $\operatorname{cl}(\operatorname{cl}(F, A))=\operatorname{cl}(F, A)$.

(4) If $L$ satisfies $a \odot \bigwedge_{i \in I} b_{i}=\bigwedge_{i \in I}\left(a \odot b_{i}\right)$, then $\operatorname{cl}((F, A) \odot(G, A)) \leq$ $\operatorname{cl}(F, A) \odot \operatorname{cl}(G, A)$. 
Proof. (1) Since $(U, A)(y, x) \odot\left(0_{X}, A\right)(x)=\left(0_{X}, A\right)(y), \operatorname{cl}\left(0_{X}, A\right)=$ $\left(0_{X}, A\right)$. Other case it is trivial.

(2) For $(U, A) \in \mathbf{U}$, since $\left(1_{\triangle}, A\right) \leq(U, A)$ from (SU4),

$$
\begin{aligned}
& \left.\bigvee_{x \in X}(U, A)(y, x) \odot(F, A)(x)\right) \\
& \geq \bigvee_{x \in X}\left(1_{\triangle}, A\right)(y, x) \odot(F, A)(x)=(F, A)(x)
\end{aligned}
$$

Hence $c l_{\mathbf{U}}^{r}(F, A) \geq(F, A)$.

(3)

$$
\begin{aligned}
& c l_{\mathbf{U}}^{r}(F, A)(y)=\bigwedge_{(U, A) \in \mathbf{U}}\left(\bigvee_{x \in X}(U, A)(y, x) \odot(F, A)(x)\right) \\
& \geq \bigwedge_{\left(U_{1}, A\right) \in \mathbf{U}}\left(\bigvee_{x \in X} \bigvee_{z \in X}\left(U_{1}, A\right)(y, z)\right. \\
& \left.\odot\left(U_{1}, A\right)(z, x) \odot(F, A)(x)\right)(\text { by }(\mathrm{SU} 5)) \\
& \geq \bigwedge_{\left(U_{1}, A\right) \in \mathbf{U}}\left(\bigvee_{z \in X}\left(U_{1}, A\right)(y, z) \odot\right. \\
& \left.\bigwedge_{\left(U_{1}, A\right) \in \mathbf{U}} \bigvee_{x \in X}\left(U_{1}, A\right)(z, x) \odot(F, A)(x)\right) \\
& =\bigwedge_{\left(U_{1}, A\right) \in \mathbf{U}}\left(\bigvee_{z \in X}\left(U_{1}, A\right)(y, z) \odot c l_{\mathbf{U}}^{r}(F, A)(z)\right) \\
& =c l_{\mathbf{U}}^{r}\left(c l_{\mathbf{U}}^{r}(F, A)\right)(y)
\end{aligned}
$$

(4)

$$
\begin{aligned}
& c l_{\mathbf{U}}^{r}((F, A) \odot(G, A))(y) \\
& =\bigwedge_{U \in \mathbf{U}}\left(\bigvee_{x \in X}(U, A)(y, x) \odot(F, A)(x) \odot(G, A)(x)\right) \\
& =\bigwedge_{U_{1} \odot U_{2} \in \mathbf{U}}\left(\bigvee_{x \in X}\left(U_{1}, A\right)(y, x) \odot\left(U_{2}, A\right)(y, x)\right. \\
& \odot(F, A)(x) \odot(G, A)(x)) \\
& \leq \bigwedge_{U_{1}, U_{2} \in \mathbf{U}}\left(\bigvee_{x \in X}\left(U_{1}, A\right)(y, x) \odot\left(U_{2}, A\right)(y, x)\right. \\
& \odot(F, A)(x) \odot(G, A)(x)) \\
& =\bigwedge_{U_{1} \in \mathbf{U}}\left(\bigvee_{x \in X}\left(U_{1}, A\right)(y, x) \odot(F, A)(x)\right) \\
& \odot \bigwedge_{U_{2} \in \mathbf{U}}\left(\bigvee_{x \in X}\left(U_{2}, A\right)(y, x) \odot(G, A)(x)\right) \\
& =c l_{\mathbf{U}}^{r}(F, A)(y) \odot c l_{\mathbf{U}}^{r}(G, A)(y) .
\end{aligned}
$$

For $c l_{\mathbf{U}}^{l}$, it is similarly proved.

Remark 11. If $(L, \odot)$ is a continuous t-norm, then $a \odot \bigwedge_{i \in I} b_{i}=\bigwedge_{i \in I}(a \odot$ $\left.b_{i}\right)$.

Theorem 12. Let $(X, A, \mathbf{U})$ be a soft quasi-uniform space and $a \odot$ $\bigwedge_{i \in I} b_{i}=\bigwedge_{i \in I}\left(a \odot b_{i}\right)$ for $a, b_{i} \in L$. Define $\tau_{\mathbf{U}}^{r}, \tau_{\mathbf{U}}^{l} \subset S(X, A)$ as follows

$$
\begin{aligned}
\tau_{\mathbf{U}}^{r} & =\left\{(F, A) \in S(X, A) \mid c l_{\mathbf{U}}^{r}(F, A)=(F, A)\right\}, \\
\tau_{\mathbf{U}}^{l} & =\left\{(F, A) \in S(X, A) \mid c l_{\mathbf{U}}^{l}(F, A)=(F, A)\right\} .
\end{aligned}
$$


Then (1) $\tau_{\mathbf{U}}^{r}$ is a soft topology on $X$ such that $\tau_{\mathbf{U}}^{r}=\left\{c l_{\mathbf{U}}^{r}(F, A) \mid(F, A) \in\right.$ $S(X, A)\}$.

(2) $\tau_{\mathbf{U}}^{l}$ is a soft topology on $X$ such that $\tau_{\mathbf{U}}^{l}=\left\{c l_{\mathbf{U}}^{l}(F, A) \mid(F, A) \in\right.$ $S(X, A)\}$.

Proof. (1) (ST1) Since $c l_{\mathbf{U}}^{r}\left(0_{X}, A\right)=\left(0_{X}, A\right)$ and $c l_{\mathbf{U}}^{r}\left(1_{X}, A\right) \geq\left(1_{X}, A\right)$, then $\left(0_{X}, A\right),\left(1_{X}, A\right) \in \tau_{\mathbf{U}}^{r}$.

(ST2) Let $(F, A),(G, A) \in \tau_{\mathbf{U}}^{r}$. Then $c l_{\mathbf{U}}^{r}(F, A)=(F, A)$ and $c l_{\mathbf{U}}^{r}(G, A)=$ $(G, A)$. It follows

$$
\begin{aligned}
c l_{\mathbf{U}}^{r}((F, A) \odot(G, A)) & \leq c l_{\mathbf{U}}^{r}(F, A) \odot c l_{\mathbf{U}}^{r}(G, A) \\
& =(F, A) \odot(G, A) .
\end{aligned}
$$

Thus $(F, A) \odot(G, A) \in \tau_{\mathbf{U}}^{r}$.

(ST3) Let $\left(F_{i}, A\right) \in \tau_{\mathbf{U}}^{r}$ for each $i \in I$. Then

$$
\begin{aligned}
& \bigwedge_{U \in \mathbf{U}}\left(\bigvee_{x \in X}(U, A)(y, x) \odot\left(F_{i}, A\right)(x)\right)=\left(F_{i}, A\right)(y) \\
& \bigwedge_{U \in \mathbf{U}}\left(\bigvee_{x \in X}(U, A)(y, x) \odot \bigvee_{i \in I}\left(F_{i}, A\right)(x)\right) \\
& =\bigwedge_{U \in \mathbf{U}} \bigvee_{i \in I}\left(\bigvee_{x \in X}(U, A)(y, x) \odot\left(F_{i}, A\right)(x)\right) \\
& =\bigvee_{i \in I} \bigwedge_{U \in \mathbf{U}}\left(\bigvee_{x \in X}(U, A)(y, x) \odot\left(F_{i}, A\right)(x)\right) \\
& \leq \bigvee_{i \in I}\left(F_{i}, A\right)(y) .
\end{aligned}
$$

By Theorem 10(2), $c_{\mathbf{U}}^{r}\left(\bigvee_{i \in I}\left(F_{i}, A\right)\right)=\bigvee_{i \in I}\left(F_{i}, A\right)$. So, $\bigvee_{i \in I}\left(F_{i}, A\right) \in \tau_{\mathbf{U}}^{r}$. Thus $\tau_{\mathbf{U}}^{r}$ is a soft topology. Put $\tau=\left\{c l_{\mathbf{U}}^{r}(F, A) \mid(F, A) \in S(X, A)\right\}$. Let $c l_{\mathbf{U}}^{r}(F, A) \in$ $\tau$. Since $c l_{\mathbf{U}}^{r}\left(c l_{\mathbf{U}}^{r}(F, A)\right)=c l_{\mathbf{U}}^{r}(F, A), c l_{\mathbf{U}}^{r}(F, A) \in \tau_{\mathbf{U}}^{r}$. Let $(F, A) \in \tau_{\mathbf{U}}^{r}$. Since $(F, A)=c l_{\mathbf{U}}^{r}(F, A),(F, A) \in \tau$. Hence $\tau=\tau_{\mathbf{U}}^{r}$.

(2) It is similarly proved in (1).

Theorem 13. Let $f_{\phi}:\left(X, A, \mathbf{U}_{X}\right) \rightarrow\left(Y, B, \mathbf{U}_{Y}\right)$ be an uniform continuous soft map. Then

(1) $f_{\phi}:\left(X, A, \tau_{\mathbf{U}_{X}}^{r}\right) \rightarrow\left(Y, B, \tau_{\mathbf{U}_{Y}}^{r}\right)$ is a continuous soft map.

(2) $f_{\phi}:\left(X, A, \tau_{\mathbf{U}_{X}}^{l}\right) \rightarrow\left(Y, B, \tau_{\mathbf{U}_{Y}}^{l}\right)$ is a continuous soft map.

(3) $f_{\phi}\left(c l_{\mathbf{U}}^{r}(F, A)\right) \leq c l_{\mathbf{U}_{Y}}^{r}\left(f_{\phi}(F, A)\right)$.

(4) $f_{\phi}\left(c l_{\mathbf{U}}^{l}(F, A)\right) \leq c l_{\mathbf{U}_{Y}}^{l}\left(f_{\phi}(F, A)\right)$. 
Proof (1) Since $(f \times f)_{\phi}^{-1}(V) \in \mathbf{U}_{X}$ for each $(V, B) \in \mathbf{U}_{Y}$, let $(G, B) \in \tau_{\mathbf{U}_{Y}}$, that is,

$$
\bigwedge_{(V, B) \in \mathbf{U}_{Y}}\left(\bigvee_{w \in Y}(V, B)(y, w) \odot(G, B)(w)\right)=(G, B)(y), \forall y \in Y,
$$

we have

$$
\begin{aligned}
& \bigwedge_{(U, A) \in \mathbf{U}_{X}}\left(\bigvee_{x \in X}(U, A)(x, z) \odot f_{\phi}^{-1}(G, B)(z)\right) \\
& \leq \bigwedge_{(f \times f)_{\phi}^{-1}(V, B) \in \mathbf{U}_{X}}\left(\bigvee_{x \in X}(f \times f)_{\phi}^{-1}(V, B)(x, z) \odot f_{\phi}^{-1}(G, B)(z)\right) \\
& \leq \bigwedge_{(f \times f)_{\phi}^{-1}(V, B) \in \mathbf{U}_{X}}\left(\bigvee_{x \in X}(V, B)(f(x), f(z)) \odot(G, B)(f(z))\right) \\
& \leq \bigwedge_{(V, B) \in \mathbf{U}_{Y}}\left(\bigvee_{x \in X}(V, B)(f(x), f(z)) \odot(G, B)(f(z))\right) \\
& \leq(G, B)(f(x))=f_{\phi}^{-1}(G, B)(x)
\end{aligned}
$$

By Theorem 10(2), $f_{\phi}^{-1}(G, B) \in \tau_{\mathbf{U}_{Y}}$.

(3)

$$
\begin{aligned}
& c l_{\mathbf{U}_{Y}}^{r}\left(f_{\phi}(F, A)\right)(y) \\
& =\bigwedge_{(V, B) \in \mathbf{U}_{Y}} \bigvee_{w \in Y}\left((V, B)(y, w) \odot f_{\phi}(F, A)(w)\right) \\
& \geq \bigwedge_{(V, B) \in \mathbf{U}_{Y}} \bigvee_{x \in X}\left((V, B)(y, f(x)) \odot f_{\phi}(F, A)(f(x))\right) \\
& \geq \bigwedge_{(V, B) \in \mathbf{U}_{Y}} \bigvee_{x \in X} \bigvee_{z \in f^{-1}(y)}\left((V, B)(f(z), f(x)) \odot f_{\phi}(F, A)(f(x))\right) \\
& \geq \bigwedge_{(V, B) \in \mathbf{U}_{Y} \bigvee_{x \in X} \bigvee_{z \in f^{-1}(y)}\left((f \times f)_{\phi}^{-1}(V, B)(z, x) \odot(F, A)(x)\right)} \\
& \left.\geq \bigvee_{z \in f^{-1}(y)} \bigwedge_{(U, A) \in \mathbf{U}_{X} \bigvee_{x \in X}((U, A)(z, x)}{ }(F, A)(x)\right)=f_{\phi}\left(c l_{\mathbf{U}}^{r}(F, A)\right)(y) .
\end{aligned}
$$

(2) and (4) are similarly proved as (1) and (3), respectively.

Lemma 14. For every $(F, A),(G, A) \in S(X, A)$, we define $\left(U_{F}, A\right) \in$ $S(X \times X, A)$ by

$$
U_{F}(a)(x, y)=F(a)(x) \rightarrow F(a)(y)
$$

then we have the following statements:

(1) $\left(1_{X \times X}, A\right)=\left(U_{0_{X}}, A\right)=\left(U_{1_{X}}, A\right)$,

(2) $\left(1_{\triangle}, A\right) \leq\left(U_{F}, A\right)$,

(3) $\left(U_{F}, A\right) \circ\left(U_{F}, A\right)=\left(U_{F}, A\right)$,

(4) $\left(U_{F}, A\right) \odot\left(U_{G}, A\right) \leq\left(U_{F \odot G}, A\right)$. 
Proof. (1)

$$
\begin{aligned}
& 1_{X \times X}(a)(x, y)=1=U_{0_{X}}(a)(x, y)=0_{X}(a)(x) \rightarrow 0_{X}(a)(y) \\
& =1_{X}(a)(x) \rightarrow 1_{X}(a)(y)=U_{1_{X}}(a)(x, y)
\end{aligned}
$$

(2) Since $U_{F}(a)(x, x)=F(a)(x) \rightarrow F(a)(x)=0,\left(1_{\triangle}, A\right) \leq\left(U_{F}, A\right)$.

(3) $\left(U_{F}, A\right) \circ\left(U_{F}, A\right) \leq\left(U_{F}, A\right)$ from

$$
\begin{aligned}
& \left(U_{F}(a) \circ U_{F}(a)\right)(x, z)=\bigvee_{y \in X}\left(U_{F}(a)(x, y) \circ U_{F}(a)(y, z)\right) \\
& =\bigvee_{y \in X}((F(a)(x) \rightarrow F(a)(y)) \odot(F(a)(y) \rightarrow F(a)(z))) \\
& \leq F(a)(x) \rightarrow F(a)(z)=U_{F}(a)(x, z) .
\end{aligned}
$$

$\left(U_{F}, A\right) \circ\left(U_{F}, A\right) \geq\left(U_{F}, A\right)$ from

$$
\begin{aligned}
& \left(U_{F}(a) \circ U_{F}(a)\right)(x, z)=\bigvee_{y \in X}\left(U_{F}(a)(x, y) \circ U_{F}(a)(y, z)\right) \\
& \geq((F(a)(x) \rightarrow F(a)(x)) \odot(F(a)(x) \rightarrow F(a)(z)))=U_{F}(a)(x, z) .
\end{aligned}
$$

(4) By Lemma 2 (12),

$$
\begin{aligned}
& U_{F}(a)(x, y) \odot U_{G}(a)(x, y) \\
& =(F(a)(x) \rightarrow F(a)(y)) \odot(G(a)(x) \rightarrow G(a)(y)) \\
& \leq(F(a)(x) \odot G(a)(x) \rightarrow F(a)(y) \odot G(a)(y) \\
& =U_{F \odot G}(a)(x, y) .
\end{aligned}
$$

Theorem 15. Let $(X, A, \tau)$ be a soft topological space. Define a function $\mathbf{U}_{\tau}: S(X \times X, A) \rightarrow L$ by

$$
\mathbf{U}_{\tau}=\left\{(U, A) \in S(X \times X, A) \mid \odot_{i=1}^{n}\left(U_{G_{i}}, A\right) \leq(U, A),\left(G_{i}, A\right) \in \tau\right\}
$$

where the first $\bigvee$ is taken over every finite family $\left\{U_{\left(G_{i}, A\right)} \mid i=1, \ldots, n\right\}$.

Then:

(1) $\mathbf{U}_{\tau}$ is a soft quasi-uniformity on $X$.

(2) $\tau \subset \tau_{\mathbf{U}_{\tau}}^{l}$.

Proof (1) (SU1) Since $\left(1_{X}, A\right) \in \tau$, there exists $\left(U_{1_{X}}, A\right) \in S(X \times X, A)$ such that $\left(U_{1_{X}}, A\right) \in \mathbf{U}_{\tau}$.

(SU2) It is trivial. 
(SU3) For $(U, A),(V, A) \in \mathbf{U}_{\tau}$, there exist two finite families $\left\{\left(F_{i}, A\right) \in \tau \mid\right.$ $\left.\odot_{i=1}^{m}\left(U_{F_{i}}, A\right) \leq(U, A)\right\}$ and $\left\{\left(G_{j}, A\right) \in \tau \mid \odot_{j=1}^{n}\left(U_{G_{j}}, A\right) \leq(G, A)\right\}$. Then $(U, A) \odot(V, A) \geq\left(\odot_{i=1}^{m}\left(U_{F_{i}}, A\right)\right) \odot\left(\odot_{j=1}^{n}\left(U_{G_{j}}, A\right)\right)$. So, $(U, A) \odot(V, A) \in \mathbf{U}_{\tau}$.

(SU4) Let $(U, A) \in \mathbf{U}_{\tau}$. Then there exists a finite family $\left\{\left(F_{i}, A\right) \in \tau \mid\right.$ $\left.\odot_{i=1}^{m}\left(U_{F_{i}}, A\right) \leq(U, A)\right\}$. Since $\left(U_{F_{i}}, A\right) \geq\left(1_{\triangle}, A\right)$ from Lemma $14(2)$,

$$
\left(1_{\triangle}, A\right) \leq \odot_{i=1}^{m}\left(U_{F_{i}}, A\right) \leq(U, A) .
$$

(SU5) Let $(U, A) \in \mathbf{U}_{\tau}$. Then there exists a finite family $\left\{\left(G_{i}, A\right) \in\right.$ $\left.\tau \mid \odot_{i=1}^{m}\left(U_{G_{i}}, A\right) \leq(U, A)\right\}$. Since $\left(U_{G_{i}}, A\right) \circ\left(U_{G_{i}}, A\right)=\left(U_{G_{i}}, A\right)$ for each $i \in\{1, \ldots, m\}$ from Lemma $14(3)$, we have $\left(\odot_{i=1}^{m}\left(U_{G_{i}}, A\right) \circ\left(\odot_{i=1}^{m}\left(U_{G_{i}}, A\right)\right) \leq\right.$ $\odot_{i=1}^{m}\left(U_{G_{i}}, A\right)$ from

$$
\begin{aligned}
& \bigvee_{y \in X}\left(\left(\odot_{i=1}^{m} U_{G_{i}}(a)(x, y)\right) \odot\left(\odot_{i=1}^{m} U_{G_{i}}(a)(y, z)\right)\right) \\
& =\bigvee_{y \in X}\left(\left(\odot_{i=1}^{m}\left(G_{i}(a)(x) \rightarrow G_{i}(a)(y)\right) \odot\left(\odot_{i=1}^{m}\left(G_{i}(a)(y) \rightarrow G_{i}(a)(z)\right)\right)\right)\right. \\
& =\bigvee_{y \in X}\left(\left(\odot_{i=1}^{m}\left(G_{i}(a)(x) \rightarrow G_{i}(a)(y)\right) \odot\left(G_{i}(a)(y) \rightarrow G_{i}(a)(z)\right)\right)\right) \\
& \leq \odot_{i=1}^{m}\left(G_{i}(a)(x) \rightarrow G_{i}(a)(z)\right) .
\end{aligned}
$$

Put $(V, A)=\odot_{i=1}^{m}\left(U_{G_{i}}, A\right)$. Then $(V, A) \in \mathbf{U}_{\tau}$ with $(V, A) \circ(V, A) \leq(U, A)$. Hence $\mathbf{U}_{\tau}$ is a soft quasi-uniformity on $X$.

(2) Let $(F, A) \in \tau$. Then $\left(U_{F}, A\right) \in \mathbf{U}_{\tau}$. Since

$$
\begin{aligned}
& \bigwedge_{U \in \mathbf{U}}\left(\bigvee_{y \in X}(U, A)(y, x) \odot(F, A)(y)\right) \\
& \leq \bigvee_{y \in X}\left(\left(U_{F}, A\right)(y, x) \odot(F, A)(y)\right) \\
& =\bigvee_{y \in X}(((F, A)(y) \rightarrow(F, A)(x)) \odot(F, A)(y)) \leq(F, A)(x)
\end{aligned}
$$

Hence $(F, A) \in \tau_{\mathbf{U}_{\tau}}^{l}$.

Theorem 16. Let $f_{\phi}:\left(X, A, \tau_{X}\right) \rightarrow\left(Y, B, \tau_{Y}\right)$ be a continuous soft map. Then $f_{\phi}:\left(X, A, \mathbf{U}_{\tau_{X}}\right) \rightarrow\left(Y, B, \mathbf{U}_{\tau_{Y}}\right)$ is an uniformly continuous soft map.

Proof. We have

$$
\begin{aligned}
& (f \times f)_{\phi}^{-1}\left(U_{G}, B\right)(a)(x, y)=\left(U_{G}, B\right)(\phi(a))(f(x), f(y)) \\
& =G(\phi(a))(f(x)) \rightarrow G(\phi(a))(f(y))=f_{\phi}^{-1}(G)(a)(x) \rightarrow f_{\phi}^{-1}(G)(a)(y) \\
& =U_{f_{\phi}^{-1}(G)}(a)(x, y) .
\end{aligned}
$$

Let $(U, B) \in \mathbf{U}_{\tau_{Y}}$. Then there exists a finite family $\left\{\left(G_{i}, B\right) \in \tau_{Y}\right.$ । $\left.\odot_{i=1}^{m}\left(U_{G_{i}}, B\right) \leq(U, B)\right\}$. 
Since $\odot_{i=1}^{m}\left(U_{G_{i}}, B\right) \leq(U, B)$, we have

$$
\begin{aligned}
& (f \times f)_{\phi}^{-1}\left(\odot_{i=1}^{m}\left(U_{G_{i}}, B\right)\right)=\odot_{i=1}^{m}(f \times f)_{\phi}^{-1}\left(\left(U_{G_{i}}, B\right)\right) \\
& =\odot_{i=1}^{m}\left(U_{f_{\phi}^{-1}\left(G_{i}\right)}, B\right) \leq(f \times f)_{\phi}^{-1}((U, B)) .
\end{aligned}
$$

So, $(f \times f)_{\phi}^{-1}((U, B)) \in \mathbf{U}_{\tau_{X}}$.

Example 17. Let $X=\left\{h_{i} \mid i=\{1, \ldots, 4\}\right\}$ with $h_{i}=$ house and $E_{Y}=$ $\{e, b, w, c, i\}$ with $e=$ expensive, $b=$ beautiful, $w=$ wooden, $c=$ creative, $i=$ in the green surroundings.

Let $(L=[0,1], \odot, \rightarrow)$ be a complete residuated lattice defined by

$$
x \odot y=x \wedge y, \quad x \rightarrow y= \begin{cases}1, & \text { if } x \leq y, \\ y, & \text { otherwise. }\end{cases}
$$

Let $X=\{x, y, z\}$ be a set and $W(e), W(b) \in S(X \times X, A)$ such that

$$
W(e)=\left(\begin{array}{ccc}
1 & 0.5 & 0.5 \\
0.7 & 1 & 0.8 \\
0.4 & 0.4 & 1
\end{array}\right) W(b)=\left(\begin{array}{ccc}
1 & 0.6 & 0.7 \\
0.4 & 1 & 0.4 \\
0.5 & 0.6 & 1
\end{array}\right)
$$

Define $\mathbf{U}=\{(U, A) \in S(X \times X, A) \mid(U, A) \geq(W, A)\}$.

(1) Since $W(e) \circ W(e)=W(e)$ and $W(b) \circ W(b)=W(b)$, $\mathbf{U}$ is a soft quasi-uniformity on $X$.

(2) We have $\tau_{\mathbf{U}}^{r}=\left\{c l_{\mathbf{U}}^{r}(F, A) \mid(F, A) \in S(X, A)\right\}$ where

$$
\begin{aligned}
& c l_{\mathbf{U}}^{r}(F, A)(e)=\left(\begin{array}{l}
F(e)(x) \vee(0.5 \wedge F(e)(y)) \vee(0.5 \wedge F(e)(z)) \\
(0.7 \wedge F(e)(x)) \vee F(e)(y) \vee(0.8 \wedge F(e)(z)) \\
(0.4 \wedge F(e)(x)) \vee(0.4 \wedge F(e)(y)) \vee F(e)(z)
\end{array}\right) \\
& c l_{\mathbf{U}}^{r}(F, A)(b)=\left(\begin{array}{l}
F(b)(x) \vee(0.6 \wedge F(b)(y)) \vee(0.7 \wedge F(b)(z)) \\
(0.4 \wedge F(b)(x)) \vee F(b)(y) \vee(0.4 \wedge F(b)(z)) \\
(0.5 \wedge F(b)(x)) \vee(0.6 \wedge F(b)(y)) \vee F(b)(z)
\end{array}\right)
\end{aligned}
$$

We have $\tau_{\mathbf{U}}^{l}=\left\{c l_{\mathbf{U}}^{l}(F, A) \mid(F, A) \in S(X, A)\right\}$ where

$$
c l_{\mathbf{U}}^{l}(F, A)(e)=\left(\begin{array}{l}
F(e)(x) \vee(0.7 \wedge F(e)(y)) \vee(0.4 \wedge F(e)(z)) \\
(0.5 \wedge F(e)(x)) \vee F(e)(y) \vee(0.4 \wedge F(e)(z)) \\
(0.5 \wedge F(e)(x)) \vee(0.8 \wedge F(e)(y)) \vee F(e)(z)
\end{array}\right)
$$




$$
c l_{\mathbf{U}}^{l}(F, A)(b)=\left(\begin{array}{l}
F(b)(x) \vee(0.4 \wedge F(b)(y)) \vee(0.5 \wedge F(b)(z)) \\
(0.6 \wedge F(b)(x)) \vee F(b)(y) \vee(0.6 \wedge F(b)(z)) \\
(0.7 \wedge F(b)(x)) \vee(0.4 \wedge F(b)(y)) \vee F(b)(z)
\end{array}\right)
$$

(3) Let $\tau=\left\{\left(0_{X}, A\right),\left(1_{X}, A\right),(F, A)\right\}$ a soft topology where

$$
\begin{gathered}
F(e)=(0.4,0.5 .0 .6), \quad F(b)=(0.7,0.4 .0 .9) \\
U_{F}(e)=\left(\begin{array}{ccc}
1 & 1 & 1 \\
0.4 & 1 & 1 \\
0.4 & 0.5 & 1
\end{array}\right) U_{F}(b)=\left(\begin{array}{ccc}
1 & 0.4 & 1 \\
1 & 1 & 1 \\
0.7 & 0.4 & 1
\end{array}\right)
\end{gathered}
$$

Define $\mathbf{U}_{\tau}=\left\{(U, A) \in S(X \times X, A) \mid(U, A) \geq\left(U_{F}, A\right)\right\}$. Since $\left(U_{F}, A\right) \circ$ $\left(U_{F}, A\right)=\left(U_{F}, A\right), \mathbf{U}$ is a soft quasi-uniformity.

We have $\tau_{\mathbf{U}_{\tau}}^{l}=\left\{c l_{\mathbf{U}_{\tau}}^{l}(G, A) \mid(G, A) \in S(X, A)\right\}$ where

$$
\begin{gathered}
c l_{\mathbf{U}_{\tau}}^{l}(G, A)(e)=\left(\begin{array}{c}
G(e)(x) \vee(0.4 \wedge G(e)(y)) \vee(0.4 \wedge G(e)(z)) \\
G(e)(x) \vee G(e)(y) \vee(0.5 \wedge G(e)(z)) \\
G(e)(x) \vee G(e)(y) \vee G(e)(z)
\end{array}\right) \\
c l_{\mathbf{U}_{\tau}}^{l}(G, A)(b)=\left(\begin{array}{c}
G(b)(x) \vee G(b)(y) \vee(0.7 \wedge G(b)(z)) \\
0.4 \wedge G(b)(x)) \vee G(b)(y) \vee(0.4 \wedge G(b)(z)) \\
G(e)(x) \vee G(e)(y) \vee G(e)(z)
\end{array}\right)
\end{gathered}
$$

Since $c l_{\mathbf{U}_{\tau}}^{l}(F, A)=(F, A), \tau \subset \tau_{\mathbf{U}_{\tau}}^{l}$.

\section{References}

[1] K.V. Babitha, J.J. Sunil, Soft set relations and functions, Compu. Math. Appl., 60 (2010), 1840-1849, doi: 10.1016/j.camwa.2010.07.014.

[2] N. Cag̈man, S. Karatas and S. Enginoglu, Soft topology, Comput. Math. Appl., 62 (1) (2011), 351-358. doi: 10.1016/j.camwa.2011.05.016.

[3] F. Feng, X. Liu, V.L. Fotea, Y.B. Jun, Soft sets and soft rough sets, Information Sciences, 181 (2011), 1125-1137, doi: 10.1016/j.ins.2010.11.004.

[4] P. Hájek, Metamathematices of Fuzzy Logic, Kluwer Academic Publishers, Dordrecht (1998), doi: 10.1007/978-94-011-5300-3.

[5] U. Höhle, S.E. Rodabaugh, Mathematics of Fuzzy Sets: Logic, Topology, and Measure Theory, The Handbooks of Fuzzy Sets Series 3, Kluwer Academic Publishers, Boston, 1999, doi: 10.1007/978-1-4615-5079-2.

[6] A.K. Katsaras, On fuzzy uniform spaces, J. Math. Anal. Appl., 101, 1984, 97-113. doi: 10.1016/0022-247x(84)90060-x. 
[7] J.M. Ko, Y.C. Kim, Soft $L$-topologies and soft $L$-neighborhood systems, (accepted to) J. Math. Comput. Sci.

[8] J.M. Ko, Y.C. Kim, Soft $L$-uniformities and soft $L$-neighborhood systems, (accepted to) J. Math. Comput. Sci.

[9] J.M. Ko, Y.C. Kim, Soft $L$-fuzzy quasi-uniformities and soft $L$-fuzzy topogenous orders, (submit to) Int. J. of Pure and Applied Math.

[10] R. Lowen, Fuzzy uniform spaces, J. Math. Anal. Appl., 82 (1981), 370-385, doi: 10.1016/0022-247x(81)90202-x.

[11] D. Molodtsov, Soft set theory, Comput. Math. Appl., 37 (1999), 19-31.

[12] Z. Pawlak, Rough sets, Int. J. Comput. Inf. Sci., 11 (1982), 341-356.

[13] Z. Pawlak, Rough probability, Bull. Pol. Acad. Sci. Math., 32 (1984), 607-615.

[14] M. Shabir, M. Naz, On soft topological spaces, Comput. Math. Appl.,61 (2011), 17861799, doi: 10.1016/j.camwa.2011.02.006.

[15] B. Tanay, M. B. Kandemir, Topological structure of fuzzy soft sets, Comput. Math. Appl., 61 (10) (2011), 2952-2957, doi: 10.1016/j.camwa.2011.03.056.

[16] Hu Zhao, Sheng-Gang Li, L-fuzzifying soft topological spaces and L-fuzzifying soft interior operators, Ann. Fuzzy Math. Inform., 5 (3) (2013), 493-503.

[17] Í. Zorlutuna, M. Akdag, W. K. Min, S. Atmaca, Remarks on soft topological spaces, Ann. Fuzzy Math. Inform., 3 (2) (2012), 171-185. 
